\title{
Molecular Basis of the 'Anti-Aging' Effect of Spermidine and Other Natural Polyamines - A Mini-Review
}

\author{
Nadège Minois \\ School of Biology, University of St Andrews, St Andrews, UK
}

\section{Key Words}

Spermidine - Polyamines - Longevity · Aging · Autophagy · Inflammation $\cdot$ Metabolism $\cdot$ Cell survival

\begin{abstract}
Background: Spermidine, a naturally occurring polyamine, has recently emerged as exhibiting anti-aging properties. Its supplementation increases lifespan and resistance to stress, and decreases the occurrence of age-related pathology and loss of locomotor ability. Its mechanisms of action are just beginning to be understood. Objectives: An up-to-date overview of the so far identified mechanisms of action of spermidine and other polyamines on aging is presented. Methods: Studies of aging and of the molecular effects of polyamines in general and spermidine in particular are used to synthesize our knowledge on what molecular mechanisms spermidine and other polyamines trigger to positively affect aging. Results: Autophagy is the main mechanism of action of spermidine at the molecular level. However, recent research shows that spermidine can act via other mechanisms, namely inflammation reduction, lipid metabolism and regulation of cell growth, proliferation and death. It is suggested that the main pathway used by spermidine to
\end{abstract}

trigger its effects is the MAPK pathway. Conclusions: Given that polyamines can interact with many molecules, it is not surprising that they affect aging via several mechanisms. Many of these mechanisms discovered so far have already been linked with aging and by acting on all of these mechanisms, polyamines may be strong regulators of aging.

(c) 2014 S. Karger AG, Basel

\section{Introduction}

Aging is a multifaceted process, probably caused by a myriad of interacting factors and with consequences at all levels of the organism. Research into the subject has revealed that factors leading to aging are as varied as sustained exposure to cellular stress, chronic inflammation, dysregulation of lipid metabolism, autophagy and cell survival and death. These factors will impinge upon each other in complex interactions. Effective interventions against aging will need to be able to impact as many as possible of the factors causing aging and their interactions. Dietary restriction may be one such intervention with its wide-ranging effects. Polyamines, especially spermidine, have also emerged as strong potential candidates

\section{KARGER}

E-Mail karger@karger.com www.karger.com/ger
(C) 2014 S. Karger AG, Basel

0304-324X/14/0604-0319\$39.50/0 
Fig. 1. Polyamine metabolism. ODC $=$ Ornithine decarboxylase; $\mathrm{PAO}=$ polyamine oxidase; $\mathrm{SMO}=$ spermine oxidase; Spd Syn $=$ spermidine synthase; Spm Syn = spermine synthase; SSAT $=$ spermidine/ spermine $\mathrm{N}^{1}$-acetyltransferase [for more details, see 1]. The levels of polyamines in many mammalian tissues are in the order spermidine $>$ spermine $>$ putrescine, with the ratios between them easily varying from 2 to 20.

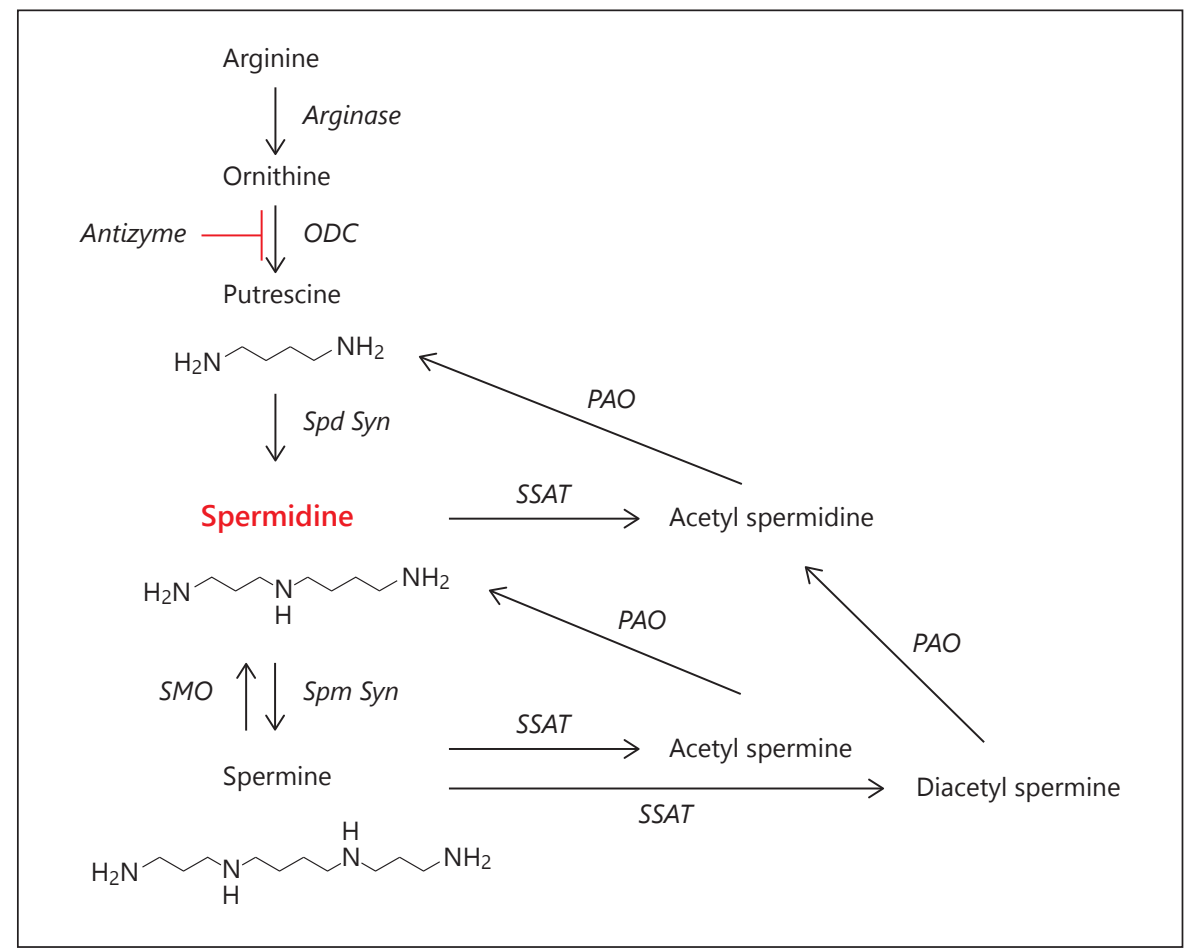

and this review will give evidence to support the involvement of polyamines in aging.

Spermidine belongs to the family of polyamines. Polyamines are polycations that will interact readily with negatively charged molecules, including DNA, RNA and lipids. This ability to bind such different molecules means polyamines are involved in many important processes such as DNA stability, cell growth, proliferation and death. To regulate these processes, a set of specific enzymes tightly regulate polyamine synthesis and catabolism (fig. 1) [reviewed in 1]. Polyamine metabolism is very dynamic and many studies have investigated the role of all three main polyamines (putrescine, spermidine and spermine) on aging, so this review will discuss all three polyamines and not only spermidine.

It has been known for some time that polyamine levels decrease with age [reviewed in 1]. However, it is only recently that the effect of polyamines, especially spermidine, on aging has been investigated. Indeed, Pucciarelli et al. [2] recently observed that spermidine levels in humans aged between 60 and 80 were lower than in humans below 50 but humans older than 90 have levels similar to people below 50 . These results suggest that maintaining spermidine levels in aging may contribute to longevity. Polyamine levels can be increased in both model organisms and humans by supplementation in food or water
[3-5], so the effect of polyamines on aging can be investigated relatively easily. Examples of food and drinks containing high levels of polyamines are rice bran, green pepper, broccoli, soybeans, mushrooms, oranges, and green tea.

We have shown that yeast cells Saccharomyces cerevisiae, nematode worms Caenorhabditis elegans, fruit flies Drosophila melanogaster as well as human peripheral blood mononuclear cells supplemented with spermidine had increased lifespans [3]. Yeast cells unable to synthesize polyamines are short-lived and spermidine addition rescued this lifespan defect [3]. Soda et al. [5, 6] also reported that a high-polyamine diet decreased mortality in mice. However in both cases, mice were sacrificed at 88 weeks of age for use in other measurements, precluding a full lifespan measurement. The same authors also showed that a high-polyamine diet decreased the incidence of age-related kidney glomerular atrophy. Aging triggered DNA methylation changes in kidneys and the high-polyamine diet suppressed these changes [6]. Finally, they reported that these mice kept thicker coat and higher activity levels with age, although these variables were not quantified [5]. Recently, we found that spermidine reduced the age-related decline of climbing activity in D. melanogaster [Minois, unpubl. results]. It was also reported that spermidine feeding re- 
duced levels of age-related oxidative damage in mice [3] and age-related overproduction of reactive oxygen species in yeast [7]. Although not measured as a function of age, spermidine has positive effects on variables related to aging, namely resistance to stress. Yeast cells fed spermidine were more resistant to heat and hydrogen peroxide [3]. We also observed that spermidine-treated D. melanogaster was more resistant to paraquat and hydrogen peroxide [8].

Evidence has accumulated that spermidine in particular and polyamines in general have implications for the aging process [1]. However, it is recently that the mechanisms of action of spermidine are being brought to light. This short review will discuss this research and give upto-date information on the molecular basis of action of polyamines in general and spermidine in particular.

\section{Autophagy}

Autophagy has been identified so far as the main mechanism of action of spermidine effects on aging. Autophagy is the main recycling mechanism of the cell, allowing the destruction and re-use of unneeded or damaged molecules or whole organelles. A deficient autophagy has been linked to many age-related diseases.

We have shown in all models tested that spermidine quickly induced autophagy [3]. This was observed in yeast, worms, flies and mouse liver cells. Furthermore, yeast cells, worms and flies deficient in autophagy did not exhibit an increased lifespan upon spermidine feeding, showing that autophagy is indispensable for the effect of spermidine on lifespan [3]. Lack of autophagy also prevented spermidine from increasing resistance to paraquat and reducing locomotor decline induced by paraquat in Drosophila [8].

The induction of autophagy by spermidine is independent of pathways previously associated with aging involving sirtuins as observed in human colon cancer HCT116 cells, yeast and worms [7]. It is also independent of the canonical mTOR pathway, as spermidine did not alter the phosphorylation status of mTOR or its substrate, ribosomal protein S6 kinase [7]. Spermidine affected the acetylation profile of many proteins, a significant proportion of them belonging to the human autophagy protein network. Spermidine mostly triggered deacetylation in the cytosol and acetylation in the nucleus. The authors reported that spermidine can induce short-term autophagy without transcription of new proteins and that autophagy by spermidine can be regulated by cytoplasmic (de)acety-

Polyamines and Aging lations. Although very important for the effects of spermidine on aging, autophagy is not the only mechanism of action involved, as for instance spermidine still increased hydrogen peroxide resistance in autophagy-deficient Drosophila [8].

\section{Inflammation}

Aging has been characterized by a chronic inflammation profile that leads to chronic damage to cells and is associated with many age-associated diseases. Polyamine levels generally increase with inflammation but whether they are more pro- or anti-inflammatory has been debated. As a matter of fact, polyamines trigger the production of anti-inflammatory cytokines while decreasing the production of pro-inflammatory ones, but at the same time, polyamine metabolism generates cytotoxic products (such as hydrogen peroxide) that can themselves cause inflammation. It seems that recent research shows that polyamines have mostly anti-inflammatory effects, some of them recently reviewed [9].

Spermidine treatment of microglial cells, the prime effector cells in inflammatory responses in the central nervous system, decreased production of pro-inflammatory mediators and cytokines in reaction to lipopolysaccharide (LPS) exposure [10]. Spermidine treatment decreased nitric oxide and prostaglandin $\mathrm{E}_{2}$ production in a dose-dependent manner, as well as the mRNA expression of pro-inflammatory cytokines, among them interleukin-6 and tumor necrosis factor- $\alpha$. The authors' work suggested that this anti-inflammatory response was triggered by the suppression of the translocation in the nucleus of NF- $\kappa \mathrm{B}$ p 65 subunit, leading to a decreased phosphorylation of Akt and MAPK.

Recently, Paul and Kang [11] studied the effect of polyamines on ear edema in mice. They induced an inflammatory response in the ear by applying 12 -O-tetradecanoylphorbol-13-acetate (TPA). Prior to TPA application, miceweretopicallytreated with saline, hydrocortisone (positive anti-inflammatory control) or the main three polyamines (putrescine, spermidine or spermine). The authors showed that application of polyamines reduced ear thickness in a dose-dependent manner to levels similar to the positive control. Spermidine and spermine induced the highest water content decrease. Polyamines also reduced neutrophil infiltration in the ear tissues. The authors then induced inflammation in mice macrophages with LPS. Polyamines decreased nitric oxide production triggered by the LPS treatment, spermine having the 
strongest effect. They also inhibited the expression of interleukin- $1 \beta$ and tumor necrosis factor- $\alpha$.

It has to be kept in mind that these studies focus on acute inflammation, in contrast with the chronic, silent inflammation observed in aging. Work on the effects of polyamines on chronic inflammation is therefore needed. However, the present research suggests that spermidine in particular and polyamines in general could promote their anti-aging properties by reducing inflammation.

\section{Lipid Metabolism}

Lipid metabolism has recently emerged as a strong regulator of health and lifespan. A dysfunction in lipid metabolism can trigger deleterious consequences on health and ultimately aging and lifespan. On the other hand, many mutations increasing lifespan have been associated with increased levels of stored lipids (TAG) and changes in lipid profiles (composition and saturation levels). The involvement of spermidine in adipogenesis combined with our results showing that spermidine alters lipid profile in fruit flies makes lipid regulation a likely contributor for the effect of spermidine on aging.

At the cellular level, the level of lipids is regulated by the differentiation of preadipocytes into mature adipocytes. Spermidine is a key factor in this process of adipogenesis. An irreversible inhibitor of polyamine synthesis, a-difluoromethylornithine (DFMO) that depletes the cells of polyamines, completely blocked adipogenesis in 3T3-L1 cells: the cells kept a fibroblast-like appearance $[12,13]$. Treatment of the cells with natural spermidine or stable methylated analogues triggered differentiation in the presence of DFMO. These cells accumulated fat as well as control cells. Spermidine analogues could also rescue the growth arrest induced by longer exposure to DFMO, although some of them not to the level of the control cells. DFMO blocked the expression of transcription factors key in preadipocytes differentiation as well as late adipocyte markers. Supplementation with natural spermidine and some of the analogues rescued the expression of these genes to allow the differentiation of preadipocytes into mature adipocytes. The molecular mechanisms of spermidine allowing adipogenesis are summarized in figure 2.

Although these last studies showed an important involvement of spermidine in adipogenesis, other studies pointed to more complex interactions between polyamines and adipogenesis. For instance, in 3T3-L1 pre- adipocytes, inhibiting spermidine synthase or spermine synthase respectively decreased and increased TAG accumulation. These inhibitions had opposite effects in mature adipocytes. Polyamine catabolism also regulates adipogenesis in these cells [14].

We have recently discovered that spermidine altered lipid metabolism in whole D. melanogaster [Minois, unpubl. results]. Spermidine supplementation increased TAG levels and altered lipid profile. We observed that spermidine supplementation changed the relative abundance of fatty acids (for instance a decline in C14:1, C14:0, C20:3 and C20:2 and an increase in C18:2 in females) and phospholipids (for instance an increase with spermidine in ethanolamine phosphate ceramide EPCd38:1, phosphatidylcholine PC32:2 and phosphatidylethanolamine PE38 in males). Spermidine also triggered changes in the ratio of saturated over unsaturated fatty acids and some ceramide phosphoethanolamine species, the main sphingolipid in invertebrates. Most of these changes are autophagy-dependent. The changes in lipids profile will modulate membrane fluidity and proneness to oxidative damage as well as signalling. How this can affect aging will need to be further studied.

\section{Cell Growth and Death}

It is undoubted that cells senesce and lose with time appropriate responses to growth and death signals. Unregulated cell growth or death will lead to tissue or organ dysfunction. However, how cellular senescence impacts whole organism aging is not fully understood and still debated. Polyamines regulate cell growth and death. The decrease in polyamine levels with age could play a part in this cellular aging phenotype and polyamine supplementation may help lessen the effects of cellular aging. Polyamines are necessary for cell growth as chemically blocking polyamine synthesis, leading to their depletion triggers growth arrest in mammalian cells, predominantly at the $G_{0} / G_{1}$ phase of the cell cycle $[15,16]$. Growth arrest was achieved by blocking translation initiation and elongation. Initiation was stopped via the increased phosphorylation of the initiation factors eIF $2 \alpha$ and 4E-BP. Elongation was inhibited as a lack of spermidine stopped the synthesis of hypusine, a peculiar amino acid synthesized exclusively from spermidine and essential for the activation of the eukaryotic initiation factor eIF5A. A genomic profiling of cells depleted of polyamines and then allowed to grow by addition of spermidine showed that not surprisingly, growth arrest 

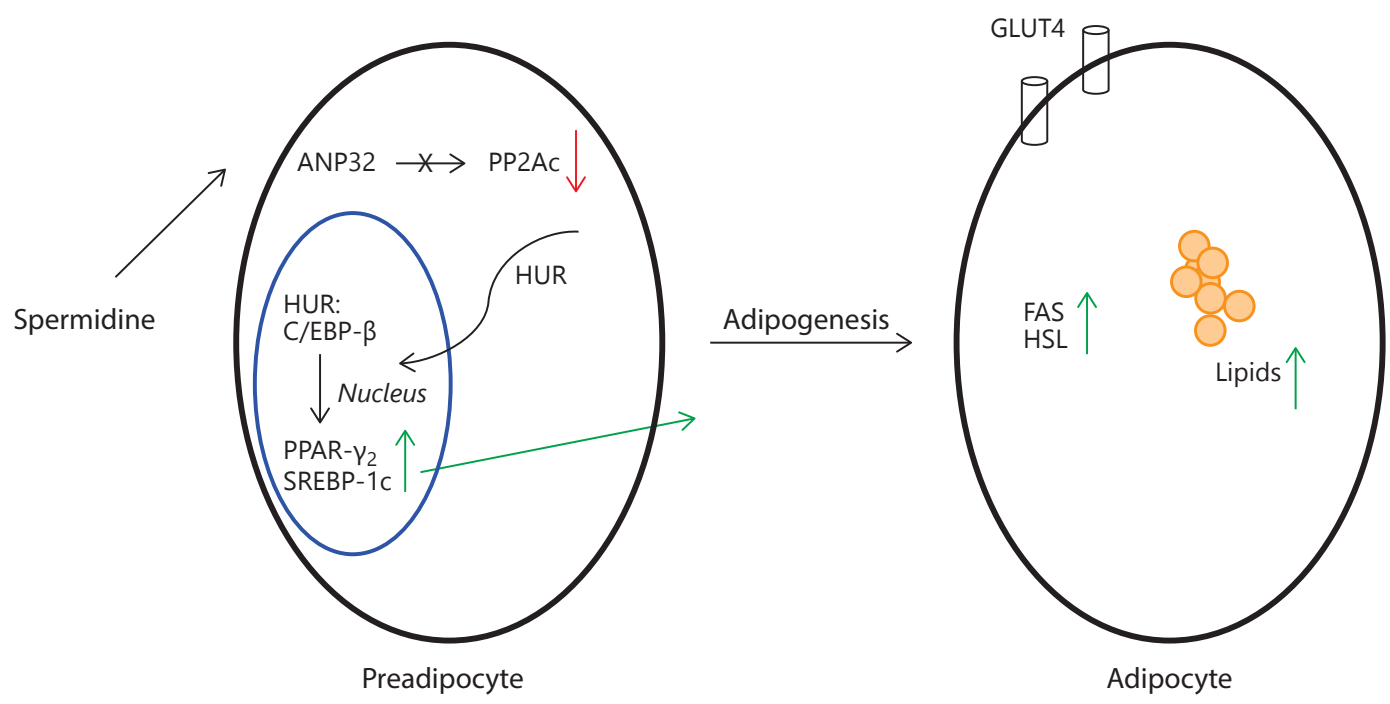

Fig. 2. Spermidine is indispensable for adipogenesis. Spermidine interacts with ANP32, blocking ANP32 interaction with HUR and PP2Ac. This decreases the phosphatase activity of PP2Ac which allows the nuclear translocation of HUR. In the nucleus, HUR binds to $\mathrm{C} / \mathrm{EBP}-\beta$, leading to the expression of transcription factors such as PPAR- $\gamma_{2}$ and SREBP-1c. Upon expression of these transcription factors, the preadipocyte differentiates into a mature adipocyte

showing increased lipid levels and expression of key markers such as FAS, HSL and GLUT4. ANP32 = Acidic nuclear phosphoproteins 32; C/EBP- $\beta=$ CCAAT/enhancer binding protein $\beta$; FAS = fatty acid synthase; GLUT4 = glucose transporter 4; HSL = hormonesensitive lipase; HUR = human antigen R; PP2Ac = protein phosphatase 2Ac; PPAR- $\gamma_{2}=$ peroxisome proliferator-activated receptor $\gamma_{2}$; SREBP-1c = sterol-regulatory element binding protein $1 \mathrm{c}$.

Fig. 3. Synthesis of the possible mechanisms of action of spermidine.

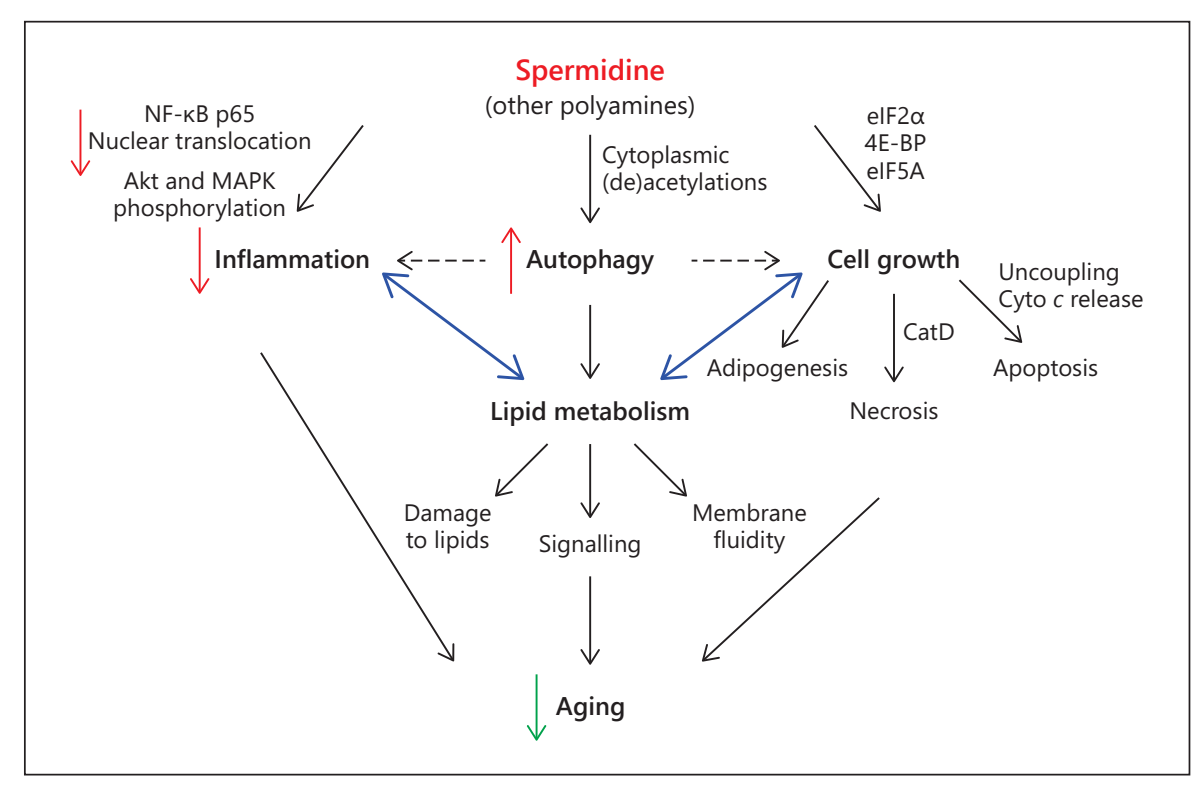

and resumption was accompanied by changes in the expression of genes linked to transcription, DNA replication and cell cycle [16]. Of the genes involved in cell cycle control Cdk7, Cdknla, Gadd45a, Mdm2 and Rb1 were upregulated and Cdk2, E2F1, PCNA, Rbl1 and
Uhrf1 were downregulated. Note that some genes activated later in the resumption of growth are associated with lipid biosynthesis. Furthermore, the authors observed that polyamine-depleted cells exhibited a change in the expression of genes known to be activated during 
cellular stress response. This stress response was rapidly stopped upon spermidine addition and cell growth resumption.

Polyamines also regulate cell death by regulating apoptosis and necrosis. The relation between polyamines and apoptosis is far from straightforward with polyamines having been reported to both induce and prevent apoptosis [reviewed in 1]. Landau et al. [16] reported that depleting NIH3T3 cells from polyamines protected them from apoptotic death. Polyamines increased $\mathrm{Ca}^{2+}$ accumulation in mitochondria, modulating the mitochondrial permeability transition and triggering apoptosis [17]. Polyamines can also directly promote cytochrome $c$ release, a prelude to apoptosis $[18,19]$. Polyamine catabolism produces some toxic by-products (such as hydrogen peroxide) that can themselves trigger uncoupling and cytochrome $c$ release [19].

Finally, polyamines can regulate the other type of cell death, necrosis. We observed that spermidine feeding increased yeast cells' chronological lifespan by reducing necrotic death and that cells unable to synthesize polyamines exhibited higher levels of necrosis [3]. A recent report by Carmona-Gutierrez et al. [20] suggested that the regulation of necrosis by polyamines may work via cathepsin D (CatD). The overexpression of PEP4, the yeast orthologue of CatD, increased chronological lifespan by reducing necrosis. This anti-necrotic effect was dependent on putrescine and spermidine synthesis, but not on spermine synthesis. Furthermore, PEP4 overexpression prevented the age-related decline of putrescine and spermidine levels.

\section{Signalling Pathways through Which Spermidine May Act}

Eventually, polyamines have to trigger their effects by activating or repressing signalling pathways at the cellular level. What can be the signalling pathway(s) by which spermidine triggers its anti-aging effects? Spermidine triggered a general hypoacetylation, which will likely induce gene expression changes [3]. Spermidine treatment changed the phosphorylation status of many kinases [7] and activated several phosphatases [21] in human colon carcinoma HCT116 cells, potentially regulating many signalling pathways. A lot of the anti-aging effects of spermidine seem to involve autophagy and pathways that will lead to the induction of autophagy are likely targets for the mechanisms of action of spermidine. Among the candidates, SIRT1, the mTOR path- way as well as AMPK are unlikely to play a role: SIRT1 is not necessary for autophagy induction by spermidine, and spermidine does not alter the phosphorylation status of mTOR, AMPK and their substrates [7]. Autophagy is also controlled by other pathways. Autophagy genes have been shown to be targets for Foxo [22], the downstream effector of the insulin-like signalling (IIS) pathway, indisputably involved in aging and lifespan regulation. Furthermore, spermidine decreases the phosphorylation status of PKB/Akt in HCT116 cells [21] and of Akt in LPS-activated BV2 microglial cells [10]. It will be interesting to study whether the effect of spermidine on lifespan overlaps with the IIS pathway. A likely candidate for regulating the anti-aging effects of spermidine is the MAPK pathway, another pathway involved in autophagy modulation [23]. In addition to regulating autophagy, it is known that the MAPK pathway interacts with polyamines [24]. CK2, a ubiquitous kinase, phosphorylates KSR (kinase suppressor of ras that serves as scaffold and can act as raf activator) and also directly raf. Polyamines change the phosphorylation of raf by CK2, either acting as inhibitor (spermine) or activator (spermine plus spermidine or putrescine) of raf. CK2 could act as a sensor for polyamine levels and translate the information to the MAPK pathway. Furthermore, some MAPKs show altered phosphorylation status upon spermidine treatment in HTC116 cells [21] and MAPKs phosphorylation is reduced by spermidine in LPS-activated BV2 microglial cells [10]. In tomato green fruits exposed to spermidine ( $1 \mathrm{mM}$ for 30 min) at high temperature, spermidine upregulates MAPK family genes [25] suggesting more involvement of polyamines with the MAPK pathway. Finally, the expression of transcription factors targets of MAPKs have been shown to be altered upon polyamines depletion and then re-addition of spermidine in NIH3T3 mouse fibroblasts [16].

\section{Conclusions and Perspectives}

It is herein reviewed that spermidine and related polyamines play a role in many molecular mechanisms involved in aging. These mechanisms are linked and their regulation by spermidine slows aging (fig. 3). Spermidine induces autophagy $[3,7]$, which dysfunction is linked to aging and age-related diseases. In turn, autophagy allows the modulation of lipid profile. The changes in lipid composition will alter membrane fluidity, its proneness to damage, especially lipid peroxidation, as well as signalling 
in which lipids are important messengers. Spermidine directly regulates cell growth by its action on initiation factors and translation. Spermidine also indirectly regulates cell growth via lipid metabolism, during adipogenesis for instance. Finally, spermidine and other polyamines can directly reduce inflammation by their action on the expression of pro- and anti-inflammatory cytokines. Spermidine also indirectly modulates inflammation via the changes it induces in lipid composition. Finally, spermidine may indirectly alter inflammation and cell growth via autophagy.

Although our knowledge on how polyamines bring about their anti-aging effects at the molecular level is expanding, a lot is still to be investigated. Spermidine alters the phosphorylation status of Akt and of some MAPKs [21]. It would be interesting to study the effects of spermidine in mutants in the IIS and MAPK pathways as well as the effect of spermidine on the expression of genes involved in these pathways. Likewise, other mechanisms have not been investigated yet. Spermidine alters acetylation levels [3]. Could it also modify meth- ylation status, especially DNA methylation, and trigger epigenetic changes?

Spermidine has been the most studied polyamine so far for its effects on aging. However, polyamine metabolism is very dynamic and a better understanding of the specific roles of each polyamine on aging is needed. For instance, spermidine feeding in flies increases endogenous levels of spermidine, but also of its precursor putrescine [3]. We should study the effects of all polyamines, on their own and in combination, as well as for instance methylated analogues. These analogues are metabolically more stable, some of them cannot be converted into other polyamines and each has different biochemical properties that could help us tease out the exact roles of polyamines in aging $[26,27]$.

\section{Acknowledgement}

The author wishes to acknowledge the important work of many authors that could not be cited due to space restriction.

\section{References}

1 Minois N, Carmona-Gutierrez D, Madeo F: Polyamines in aging and disease. Aging 2011; 3:716-732.

$>2$ Pucciarelli S, Moreschini B, Micozzi D, De Fronzo GS, Carpi FM, Polzonetti V, Vincenzetti S, Mignini F, Napolioni V: Spermidine and spermine are enriched in whole-blood of nona/centenarians. Rejuv Res 2012;15:590595.

$>3$ Eisenberg T, Knauer H, Schauer A, Büttner S, Ruckenstuhl C, Carmona-Gutierrez D, Ring J, Schroeder S, Magnes C, Antonacci L, Fussi H, Deszcz L, Hartl R, Schraml E, Criollo A, Megalou E, Weiskopf D, Laun P, Heeren G, Breitenbach M, Grubeck-Loebenstein B, Fahrenkrog B, Fröhlich KU, Sinner F, Tavernarakis N, Minois N, Kroemer G, Madeo F: Induction of autophagy by spermidine promotes longevity. Nat Cell Biol 2009;11:13051314.

4 Soda K, Kano Y, Sakuragi M, Takao K, Lefor A, Konishi F: Long-term oral polyamine intake increases blood polyamines concentrations. J Nutr Sci Vitaminol 2009;55:361-366.

5 Soda K, Dobashi Y, Kano Y, Tsujinaka S, Konishi F: Polyamine-rich food decreases age-associated pathology and mortality in aged mice. Exp Gerontol 2009;44:727-732.

-6 Soda K, Kano Y, Chiba F, Koizumi K, Miyaki $\mathrm{Y}$ : Increased polyamine intake inhibits ageassociated alteration in global DNA methylation and 1,2-dimethylhydrazine-induced tumorigenesis. PLoS One 2013;8:e64357.
7 Morselli E, Mariño G, Bennetzen MV, Eisenberg T, Megalou E, Schroeder S, Cabrera S, Bénit P, Rustin P, Criollo A, Kepp O, Galluzzi L, Shen S, Malik SA, Maiuri MC, Horio Y, López-Otín C, Andersen JS, Tavernarakis N, Madeo F, Kroemer G: Spermidine and resveratrol induce autophagy by distinct pathways converging on the acetylproteome. J Cell Biol 2011;192:615-629.

-8 Minois N, Carmona-Gutierrez D, Bauer MA, Rockenfeller P, Eisenberg T, Brandhorst S, Sisgrist SJ, Kroemer G, Madeo F: Spermidine promotes stress resistance in Drosophila melanogaster through autophagy-dependent and -independent pathways. Cell Death Dis 2012;3:e401.

$\checkmark 9$ Bjelaković G, Stojanović I, Jevtović Stoimenov T, Pavlović D, Kocić G, Rossi S, Tabolacci C, Nikolić J, Sokolović D, Bjelakovic LJ: Metabolic correlations of glucocorticoids and polyamines in inflammation and apoptosis. Amino Acids 2010;39:29-43.

10 Choi YH, Park HY: Anti-inflammatory effects of spermidine in lipopolysaccharidestimulated BV2 microglial cells. J Biomed Sci 2012;19:31.

11 Paul S, Kang SC: Natural polyamine inhibits mouse skin inflammation and macrophage activation. Inflamm Res 2013;62:681-688.

12 Vuohelainen S, Pirinen E, Cerrada-Gimenez M, Keinänen TA, Uimari M, Khomutov AR, Jänne J, Alhonen L: Spermidine is indispensable in differentiation of 3T3-L1 fibroblasts to adipocytes. J Cell Mol Med 2010;14:16831692.

13 Hyvönen MT, Koponen T, Weisell J, Pietilä M, Khomutov AR, Vepsäläinen J, Alhonen L, Keinänen TA: Spermidine promotes adipogenesis of 3T3-L1 cells by preventing interaction of ANP32 with HuR and PP2A. Biochem J 2013;453:467-474.

14 Ishii I, Ikeguchi Y, Mano H, Wada M, Pegg AE, Shirahata A: Polyamine metabolism is involved in adipogenesis of 3T3-L1 cells. Amino Acids 2012;42:619-626.

15 Landau G, Bercovich Z, Park MH, Kahana C: The role of polyamines in supporting growth of mammalian cells is mediated through their requirement for translation initiation and elongation. J Biol Chem 2010;285:12474-12481.

16 Landau G, Ran A, Bercovich Z, Feldmesser E, Horn-Saban S, Korkotian E, Jacob-Hirsh J, Rechavi G, Ron D, Kahana C: Expression profiling and biochemical analysis suggest stress response as a potential mechanism inhibiting proliferation of polyamine-depleted cells. J Biol Chem 2012;287:35825-35837.

17 Salvi M, Toninello A: Effects of polyamines on mitochondrial $\mathrm{Ca}^{2+}$ transport. Biochim Biophys Acta 2004;1661:113-124.

18 Stefanelli C, Stanić I, Zini M, Bonavita F, Flamigni F, Zambonin L, Landi L, Pignatti C, Guarnieri C, Caldarera CM: Polyamines directly induce release of cytochrome $c$ from heart mitochondria. Biochem J 2000;347: 875-880. 
19 Maccarrone M, Bari M, Battista N, Di Rienzo M, Falciglia K, Finazzi Agrò A: Oxidation products of polyamines induce mitochondrial uncoupling and cytochrome $c$ release. FEBS Lett 2001;507:30-34.

20 Carmona-Gutierrez D, Bauer MA, Ring J, Knauer H, Eisenberg T, Büttner S, Ruckenstuhl C, Reisenbichler A, Magnes C, Rechberger GN, Birner-Gruenberger R, Jungwirth H, Fröhlich KU, Sinner F, Kroemer G, Madeo F: The propeptide of yeast cathepsin D inhibits programmed necrosis. Cell Death Dis 2011;2:e161.
21 Bennetzen MV, Marino G, Pultz D, Morselli E, Faergeman NJ, Kroemer G, Andersen JS: Phosphoproteomic analysis of cells treated with longevity-related autophagy inducers. Cell Cycle 2012;11:1827-1840.

22 Rajeeve V, Pearce W, Cascante M, Vanhaesebroeck B, Cutillas PR: Polyamine production is downstream and upstream of oncogenic PI3K signalling and contributes to tumour cell growth. Biochem J 2013;450:619-628.

23 Vellai T, Takács-Vellai K, Sass M, Klionsky DJ: The regulation of aging: does autophagy underlie longevity? Trends Cell Biol 2009;19: 487-494.

24 Wang J, Whiteman MW, Lian H, Wang G, Singh A, Huang D, Denmark T: A non-canonical MEK/ERK signalling pathway regulates autophagy via regulating Beclin 1. J Biol Chem 2009;284:21412-21424.
25 Stark F, Pfannstiel J, Klaiber I, Raabe T: Protein kinase CK2 links polyamine metabolism to MAPK signaling in Drosophila. Cell Signal 2011;23:876-882.

-26 Cheng L, Sun RR, Wang FY, Peng Z, Kong FL, Wu J, Cao JS, Lu G: Spermidine affects the transcriptome responses to high temperature stress in ripening tomato fruit. J Zhejiang Univ Sci B 2012;13:283-297.

27 Keinänen TA, Hyvönen MT, Alhonen L, Vepsäläinen J, Khomutov AR: Selective regulation of polyamine metabolism with methylated polyamine analogues. Amino Acids 2013, Epub ahead of print. 